Bioscientia Medicina: Journal of Biomedicine \& Translational Research

Journal Homepage: www.bioscmed.com

\title{
Correlation Epidermal Growth Factor Receptor Mutation with Non-small Cell Lung Cancer in Passive Smokers: A Review
}

\section{Yuda Nabella Prameswari ${ }^{1,2^{\star}}$, Dwi Anita Suryandari ${ }^{3}$}

${ }^{1}$ Master's Programme in Biomedical Science, Faculty of Medicine, Universitas Indonesia, Jakarta, Indonesia

${ }^{2}$ Faculty of Medicine, Universitas Sultan Ageng Tirtayasa, Cilegon, Indonesia

${ }^{3}$ Department of Medical Biology, Faculty of Medicine, Universitas Indonesia, Jakarta, Indonesia

\author{
A R T I C L E I N F O \\ Keywords: \\ Epidermal growth factor receptor \\ Passive smokers \\ Non-small cell lung cancer \\ Literature review \\ Glycoprotein
}

\section{*Corresponding author: \\ Yuda Nabella Prameswari \\ E-mail address: \\ yuda.nabella@ui.ac.id}

All authors have reviewed and approved the final version of the manuscript.

https://doi.org/10.37275/bsm.v6i4.484

\begin{abstract}
A B S T R A C T
Epidermal growth factor receptor (EGFR) is a transmembrane glycoprotein with an extracellular epidermal growth factor binding domain and an intracellular tyrosine kinase domain through signaling pathways to regulate cellular proliferation. Epidermal growth factor receptor binding to its ligand will result in autophosphorylation by intrinsic tyrosine kinase activity, thereby triggering multiple signal transduction cascades. Constant or sustained activation of these downstream target sequences is thought to result in a more aggressive tumor phenotype. Mutations in EGFR are associated with non-small cell lung cancer in passive smokers.
\end{abstract}

\section{Introduction}

Indonesia is one of the countries with the thirdhighest number of smokers in the world, after China and India. ${ }^{1}$ A 2015 study by the Research and Development Agency reported that Indonesia accounts for more than 230,000 deaths due to the consumption of tobacco products each year. ${ }^{2}$ Smoking prevalence in Indonesia is $29.3 \%$ and 13 out of 33 provinces have smoking prevalence above the national average. ${ }^{2}$ As a result, more people are exposed to or inhaled cigarette smoke exhaled by active smokers. Several studies explain that passive smokers are more susceptible to respiratory tract diseases than active smokers. ${ }^{3}$ Riskesdas reported in 2018 the prevalence of passive smoking in a closed room, which was $32.4 \%$ exposed every day and $43.1 \%$ exposed only occasionally. ${ }^{2}$

Cancer is an abnormal cell that divides without control, is invasive, and can invade surrounding tissues. ${ }^{3}$ Cancer cells can spread to other parts of the body through the blood and lymph system. ${ }^{3}$ Lung cancer is one of the most important causes of cancer 
death in Indonesia. ${ }^{2}$ Data from the WHO International Agency for Research of Cancer, GLOBOCAN stated that in 2020 there were 34,783 new cases of lung cancer, ranking first of the total cancer deaths in Indonesia, which was $13.2 \% .^{4}$ Based on data from the Persahabatan Central General Hospital, 87\% of lung cancer cases are related to smoking. ${ }^{2}$

In the early stages, lung cancer does not cause specific symptoms. 5 Generally more than $60 \%$ of patients are diagnosed in an advanced state due to the rapid growth of cancer cells. About $80-85 \%$ of all lung cancers are non-small cell lung cancer (NSCLC) and 15\% are small cell lung cancer (SCLC).5,6 The most common type of NSCLC is adenocarcinoma, which accounts for $40 \%$ of all lung cancer cases. These cells are derived from type II alveolar cells that secrete mucus and other substances. ${ }^{6}$ Adenocarcinoma is the most common type of lung cancer that occurs in all people.5.6 Cancers located in the central bronchi will cause symptoms of cough, hemoptysis, wheezing, stridor, shortness of breath, and pneumonitis, while cancers located in the periphery cause chest pain, cough, shortness of breath, and symptoms of lung abscess due to cavitation of the cancer mass. 6,7 Clinical diagnosis can be identified by sputum cytology examination as a histological examination. ${ }^{7}$ If the nodule is centrally located bronchoscopy for biopsy and the mass is peripherally located transthoracal biopsy or fine-needle aspiration together with ultrasonography, CT scan, or fluoroscopy. ${ }^{7}$

Smoking-associated lung cancer occurs through the gradual accumulation of various mutations, resulting in the transformation of benign progenitor cells in the lung organs into neoplastic cells that have all the hallmarks of cancer. ${ }^{5.8}$ Lung cancer cells will deregulate growth signals and cause overexpression of growth signal receptors so that cancer cells proliferate continuously. 8

Epidermal growth factor receptor (EGFR) is a transmembrane protein with cytoplasmic kinase activity that transduces growth factor signals from the extracellular environment to the cell.9,10 One study has shown that EGFR is frequently hyperactivated in human cancers via somatic mutation and overexpression that is associated with lung cancer development.10 Lung cancer has been the most common cause of cancer death worldwide in recent decades and exhibits a five-year lower survival rate than other cancers. Treatment has evolved from surgery combined with chemotherapy as targeted therapy. This paper is used to determine the role of EGFR mutations in the diagnosis of lung cancer in passive smokers and the management of NSCLC therapy.

\section{The difference between passive smokers and active smokers}

Passive smokers are non-active smokers who inhale second-hand smoke. Second-hand smoke consists of a mixture of sidestream smoke and mainstream smoke exhaled by an active smoker. Second-hand smoke will be diluted when sidestream smoke and mainstream smoke mix with air and the concentration of tobacco smoke components will settle on the surface of the room to form transformed chemically. ${ }^{14}$

Active smokers inhale mainstream smoke, which is smoke drawn directly through the tip of the cigarette. Mainstream smoke is inhaled without dilution and smokers will receive many toxic compounds in high doses. Typically, a smoker takes 10-12 puffs from each cigarette or about 240 puffs per day for a smoker who smokes 1 pack of cigarettes daily. Smoking patterns over a long period can cause the cumulative dose of toxic components in the body to be very high. ${ }^{14}$

\section{Types of molecules inhaled by passive smokers}

Second-hand smoke, also known as environmental tobacco smoke, is a mixture of two clouds of smoke that come from burning tobacco, namely mainstream tobacco smoke and side stream tobacco smoke. ${ }^{14.15} \mathrm{~A}$ non-smoker who inhales second-hand smoke consumes nicotine and other toxic chemicals in the same way as a smoker, so the more second-hand smoke is inhaled, the higher the levels of harmful substances that settle in the respiratory system. ${ }^{15}$

Active smokers will inhale the main smoke (mainstream tobacco smoke) during inhalation, while passive smokers inhale the smoke produced from the 
end of a lit cigarette (side stream tobacco smoke) and smoke exhaled by active smokers (exhaled mainstream tobacco smoke). ${ }^{15}$ Mainstream tobacco smoke and side stream tobacco smoke are qualitatively similar in contaminants, i.e., they have fast dilution and dispersion into the room but are quantitatively different. Therefore, the ratio of side stream tobacco smoke to mainstream tobacco smoke varies according to the concentration of the constituents. This causes the physicochemical characteristics of second-hand smoke to differ between mainstream tobacco smoke and side stream tobacco smoke. ${ }^{15,16}$

Cigarette smoke is divided into two phases, namely the tar phase and the gas phase. The tar or particulate phase is a material that is trapped in the fiber filter when the smoke stream exits, so it can hold $99.9 \%$ of all particulate matter $>0.1 \mathrm{~m}$ in size, while the gas phase is a material that can pass through the filter stream. The tar phase of cigarette smoke contains $>1017$ free radicals per gram, and the gas phase contains $>1015$ free radicals per puff. Radicals associated with the tar phase have a longer lifespan than radicals associated with the gas phase. 16

Second-hand smoke or environmental tobacco smoke is produced from $85 \%$ of side stream smoke and $15 \%$ of mainstream smoke exhaled by active smokers. Side stream smoke contains a higher toxic gas component than mainstream smoke. Of all the constituents contained in cigarette smoke, nicotine is a component of the tar phase which has addictive substances. 16

\section{Correlation between passive smokers and the occurrence of lung cancer}

Mainstream tobacco smoke causes \% of several constituents of smoke which are hydrophilic and hydrophobic (except carbon monoxide) to produce particles that settle on the surface of the room and combine with gasses in the air to form compounds that cause lung cancer. As a result, the reactive components contained in mainstream tobacco smoke will be inhaled and cause a non-smoker who is exposed to environmental tobacco smoke to experience coagulation of small particles through the ventilation system which can form toxic aggregates in the lungs. Mainstream smoke inhaled by passive smokers is an aerosol containing about $1 \times 10^{10}$ potential carcinogenic particles per milliliter with a diameter of 0.1-1.0 m (0.2 $\mathrm{m}$ average diameter), including polycyclic aromatic hydrocarbons (PAHs), aromatic amines, Nnitrosamines, and other organic and inorganic compounds, such as benzene, vinyl chloride, arsenic, and chromium. PAHs and nitrosamines require metabolic activation to be carcinogenic and can penetrate deep into the lungs causing all particles to be deposited in the bronchioles and alveoli. The metabolic detoxification process of the compound will occur, so the imbalance between activation and detoxification can affect the risk of lung cancer.17.18 These events can result in inflammation and the release of components, including nicotine, which cross the lung lining and enter the circulation. Reactive gasses in tobacco smoke, such as formaldehyde are expelled in the upper airways, while insoluble and unreactive gasses, such as carbon monoxide, enter the alveoli to diffuse and are generally distributed into the blood circulation. ${ }^{17,18}$ The benzo[a]pyrene component directly enters the cells lining the upper airways and the airways in the lungs.19,20 Several other components undergo metabolic transformation into active forms and some studies report evidence that there are metabolic determinants of genes that can influence susceptibility to tobacco smoke as a cause of lung cancer. Radioactive materials, such as radon and its decay products, bismuth, and polonium, are also present in tobacco smoke. ${ }^{19}$

The International Agency for Research on Cancer (IARC) has identified 50 carcinogens in tobacco smoke. 4 An important substance causing lung cancer is tobacco-specific nitrosamine (TSNA) which is formed by nitrosation of nicotine during tobacco processing and smoking. ${ }^{19}$ Several studies suggest that eight TSNAs have been identified, including 4-(methylnitrosamino)1-(pyridyl)-1-butanone (NNK) causing lung adenocarcinoma. ${ }^{15}$ TSNAs have been associated with cancers of the esophagus, bladder, pancreas, oral cavity, and larynx. NNK compounds are the most important cause of lung cancer because they have a 
carcinogenic effect with topical and systemic administration. ${ }^{15,16} \mathrm{NNK}$ will enter directly into the lungs by inhaling tobacco smoke and NNK is absorbed systemically and hematogenous through the pulmonary circulation. ${ }^{16,18}$

The dose of smoke constituents received depends not only on the cigarette itself, but also on the duration and intensity of inhalation, the presence and competence of the filter, and the duration of cooling of the smoke before inhalation. ${ }^{20}$ the main factor that determines the intensity of cigarette use is nicotine dependence on smokers to smoke more intensively with higher inhalations per minute and deeper inhalations to meet their nicotine needs so that the peripheral bronchi in the lungs will be exposed to smoke containing carcinogens compared to the major bronchi.20 Therefore, the peripheral bronchi do not have a protective epithelium. This event causes carcinogenic TSNA to attach to the peripheral bronchi and induces lung adenocarcinoma. 16,19,20

NNK is associated with DNA mutations resulting in the activation of the oncogene KRAS and EGFR. Activation of the KRAS oncogene has been detected in $24 \%$ of human lung adenocarcinomas and is present in lung adenocarcinomas in ex-smokers. In addition, certain chemical constituents of tobacco smoke, namely benzo[a]pyrene metabolite, can damage various p53 tumor suppressor gene loci which are known to be abnormal in about $60 \%$ of primary lung cancer cases. Polycyclic aromatic hydrocarbons (PAHs) found in tobacco smoke may also target gene mutation points for lung cancer. $16,19,20$

\section{Lung cancer}

Lung cancer is one of the most important causes of cancer death in Indonesia. The term lung cancer is used for tumors originating from the respiratory epithelium, namely the bronchi, bronchioles, and alveoli. Data from the WHO International Agency for Research of Cancer, GLOBOCAN stated that in 2020 there were 34,783 new cases of lung cancer, first ranked of the total cancer deaths in Indonesia of $13.2 \% .^{3}$

Generally, most patients are diagnosed with an advanced condition because of the rapid growth of cancer cells, so patients will have a low life expectancy and only need to improve their quality of life. Clinical manifestations in lung cancer patients include weakness or malaise, anemia, weight loss, clubbing fingers. ${ }^{5}$ Cancer located in the central bronchus will cause symptoms of cough, hemoptysis, wheezing, stridor, shortness of breath, and pneumonitis. Cancer located in the periphery causes signs of chest pain, cough, shortness of breath, and symptoms of lung abscess due to cavitation of the cancer mass. ${ }^{5}$ Spread of lung cancer in the chest cavity causes tracheal obstruction, dysphagia, hoarseness, Horner's syndrome, vena cava syndrome, pleural effusion, and respiratory failure. ${ }^{5}$

Clinical diagnosis can be known by sputum cytology examination as a histological examination. If the location of nodule is central, bronchoscopy can be performed for biopsy and the mass is located at the periphery, a transthoracic biopsy or fine-needle aspiration may be performed along with ultrasonography, CT scan, or fluoroscopy. ${ }^{5}$

\section{Etiology of lung cancer}

Tobacco use is a major risk factor for lung cancer. WHO estimates that deaths from lung cancer worldwide will continue to increase as a result of increasing tobacco use globally, especially in Asia. There are currently about 1.1 billion smokers worldwide, and if this continues, that number will increase to 1.9 billion by 2025. Other risk factors are exposure to secondhand smoke, presence of lung or airway disease, hormonal factors, metastases from previous cancers, and family history of cancer. All these factors are thought to contribute to the incidence of lung cancer, but a lesser extent than smoking. ${ }^{21}$

In 1964, the US Public Health Service published a landmark report from the Surgeon General on smoking and its effects on health. The report states, (1) Smoking is associated with a $70 \%$ increase in mortality by age in men and a lower increase in mortality in women. (2) Smoking is causally associated with lung cancer in men. The magnitude of the effect of smoking is proven to be higher than all other factors that cause lung 
cancer. The risk of lung cancer increases with the length of smoking and the number of cigarettes smoked per day. ${ }^{19}$ The report estimates that the average male smoker has a 9-10-fold risk of developing lung cancer, while heavy smokers have a 20-fold risk. (3) Smoking is a very important risk factor compared to occupational exposure to chemical compounds as a cause of lung cancer in the general population. ${ }^{21}$

The influence of other carcinogens related to occupational exposures may also be responsible for the development of lung cancer. Workers exposed to asbestos and inhalation of dust containing arsenic, chromium, nickel or vinyl chloride and radon that interact synergistically with tobacco cigarettes in active smokers will increase the risk of developing lung cancer by about 55 times, while passive smokers who are exposed to asbestos exposure can increase the risk of lung cancer by 5 times. ${ }^{21}$

\section{Non-small cell lung cancer}

In general, lung cancer is grouped into non-small cell lung cancer (NSCLC) and small cell lung cancer (SCLC). NSCLC will be grouped into squamous cell carcinoma, adenocarcinoma, and large cell carcinoma, while SCLC is classified histologically into a pure small cell, mixed small cell, and large cell carcinoma.6,7 NSCLC is the most common type, which is about $80 \%-$ $85 \%$ of all lung cancer cases. Squamous cell carcinoma accounts for $25 \%-30 \%$ of all lung cancer cases. These cells arise from squamous epithelial cells in the middle bronchi of the lungs. This subtype of cancer is related to smoking habits. The most common type of NSCLC is adenocarcinoma, which accounts for $40 \%$ of all lung cancer cases. These cells are derived from type II alveolar cells that secrete mucus and other substances. Adenocarcinoma is the most common type of lung cancer that occurs in all people and grows at the edge of the lung. Type of SCLC is more aggressive than NSCLC and often has distant metastases, especially to bone, liver, adrenal glands, pericardium, brain, and spinal cord. 6.7

\section{Pathogenesis of lung cancer}

Smoking-associated lung cancer occurs through the gradual accumulation of various mutations, resulting in the transformation of benign progenitor cells in the lung organs into neoplastic cells that have all the hallmarks of cancer. Lung cancer cells will experience deregulation of growth signals and cause overexpression of growth signal receptors so that cancer cells proliferate continuously. Several growth factors and receptors are encoded by proto-oncogenes, one of which is KRAS, which is an important part of signal transduction pathways. Point mutations in the KRAS gene are detected in 15\%-20\% of all NSCLC and about $30 \%$ of lung adenocarcinomas have mutations at codon 12. Mutations in KRAS are associated with smoking activity.7,22

Receptors of growth factor tyrosine kinase, namely EGFR and HER-2 genes will be overexpressed in NSCLC. Somatic mutations of the EGFR and HER-2 genes have been reported in patients with lung adenocarcinoma who have never smoked, women, and of East Asian ethnicity. EGFR gene mutations have been detected in $40 \%$ of lung adenocarcinomas and occur in only $3 \%$ of other histologic features of NSCLC. ${ }^{19}$ The most frequently detected mutation of the EGFR gene in non-smoking lung cancer patients is an exon 19 or exon 21 deletion. HER-2 is detectable in approximately $30 \%$ of NSCLC, and is significantly more common in passive smokers by $3.2 \%$ and patients with adenocarcinoma, by $2.8 \% .^{7,22}$

In normal cells, there is a tumor suppressor gene (TSG) which plays a role in regulating cell growth activity. The most common tumor suppressor genes found in lung cancer are TP53 (17p13), retinoblastoma (13q12), P16 (9p21), and phosphatase and tensin homolog (PTEN) (10q22). The TP53 gene encodes a protein that functions as a transcription factor to detect and bind to damaged DNA. Chromosome sequence 17 p13 which is the locus of the TP53 gene, about $65 \%$ was found to be missing in NSCLC. 22,23

NSCLC is associated with an immunosuppressive tumor microenvironment that is conducive to tumor growth. NSCLC has been shown to contain large numbers of regulatory $\mathrm{T}$ cells that express high levels of cytotoxic T-lymphocyte-associated antigen-4 (CTLA4) on their surface and directly inhibit $T$ cell 
proliferation. In NSCLC there are CD8+ T cells that play a role in tumor infiltration. increased expression of programmed cell death protein 1 (PD-1), thus indicating impaired immune function. Increased expression of programmed death-ligand 1 (PD-L1) was also found in NSCLC tumor cells and was associated with suppression of dendritic cell maturation, leading to impaired immune activity in the tumor microenvironment. 22,23

\section{The progressiveness of lung cancer}

Lung cancer is a dangerous lesion that in most cases has spread before causing symptoms, so it cannot be resected. However, once other symptoms have occurred, such as hoarseness, chest pain, superior vena cava syndrome, pericardial or pleural effusion, persistent segmental atelectasis, or pneumonitis, the prognosis is poor. Lung cancer can also be identified through symptoms originating from metastases to various organs. In general, non-small cell lung cancer (NSCLC) which includes squamous cell carcinoma, adenocarcinoma, and large cell carcinoma has a better prognosis, than small cell lung cancer (SCLC). If squamous cell carcinoma or adenocarcinoma is detected before metastases or local spread occurs, a cure can be achieved by lobectomy or pneumonectomy. Currently, various therapies are available to target specific oncoproteins found in various subtypes of nonsmall cell lung cancer. Unresectable adenocarcinomas are associated with target mutations in tyrosine kinases, such as the EGFR gene that responds well to specific inhibitors. 6,24

\section{EGFR gene}

The EGFR gene provides instructions for making a receptor protein called epidermal growth factor receptor (EGFR). The protein encoded by the EGFR gene is a transmembrane glycoprotein that is a member of the protein kinase superfamily. ${ }^{25}$ EGFR is a cell surface protein that binds to epidermal growth factor. ${ }^{26}$ Binding of proteins to ligands induces receptor dimerization and autophosphorylation of tyrosine by intrinsic tyrosine kinase activity leading to cellular proliferation. Mutations in EGFR are associated with several types of lung cancer. ${ }^{26}$

\section{Structure of the EGFR gene}

The human EGFR gene is located on chromosome 7 at position $7 \mathrm{p} 11.2$ with a size of about $200 \mathrm{~kb}$ of genomic DNA. The EGFR gene consists of 28 exons and 27 introns and encodes a $170 \mathrm{kDa}$ glycoprotein which has three domains, namely the extracellular domain (exons 1-16), the spanning (exon 17), and the intracellular domain (exons 18-28). The extracellular region of the EGFR family contains two homologous ligand-binding domains (domains I and III) and two cysteine-rich domains (domains II and IV), while the intracellular domain is regulated by the $\mathrm{C}$-terminal and TK domains. ${ }^{27}$ EGFR receptor tyrosine kinase has four members called the ErbB family, consisting of EGFR, ErbB2 (HER-2 or Neu), ErbB3 (HER-3), and ErbB4 (HER-4).

Each ErbB receptor consists of a large extracellular region, transmembrane (TM) intracellular juxtamembrane $\mathrm{JM}$ ) the region, a tyrosine kinase domain, and a $\mathrm{C}$-terminal regulatory region. Ligands that regulate ErbB receptors can be separated into two main groups, namely EGFR agonists that activate EGFR, and neuregulin (NRG) that binds ErbB3 and ErbB4. 27,28

\section{Correlation between EGFR mutations and passive smokers}

Mutations in the EGFR domain tyrosine kinase (TK) are the first genetic mutations that are often found in lung cancer patients who are not smokers. ${ }^{29}$ Mutations, amplification, or overexpression of growth factor receptors such as the EGFR gene are most common in the NSCLC strain of second-hand smoke. All genetic changes have been observed in general in adenocarcinoma, a non-smoker, female, and of Asian ethnicity. gene EGFR mutations or multiple mutations can be identified during sequencing at exons 18-21 of the EGFR gene of a secondhand smoker. ${ }^{29,30}$

The EGFR kinase domain has 2 lobes, namely the $\mathrm{N}$-lobe and C-lobe, which are separated by an ATPbinding gap. The N-lobe of the EGFR kinase domain is mainly composed of regulatory -strands and C-helix, 
whereas the larger C-lobe is mostly helical and the activation loop, i.e., the A-loop. The inactive state of the wild-type (WT) EGFR is characterized by outward rotation of the C-helix stabilized by helical turns in the N-terminal portion of the A-loop. ${ }^{30,31}$

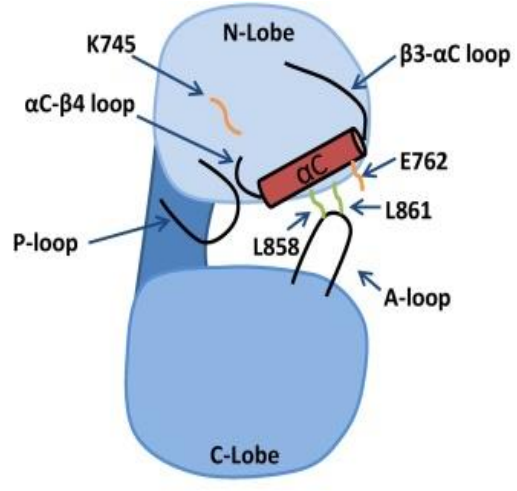

Inactive Conformation

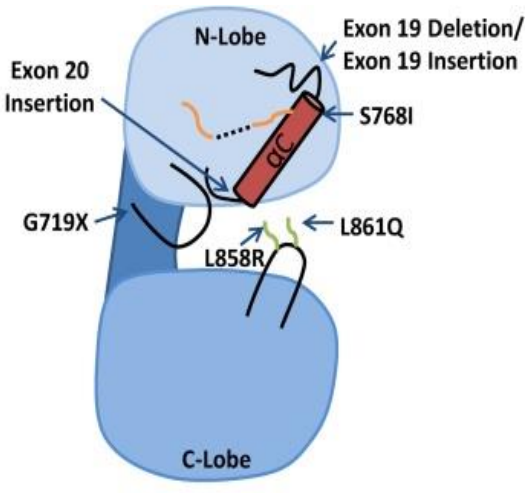

Active Conformation

Figure 1. Domain structure of inactive and active conformational EGFR genes. ${ }^{31}$

The most common EGFR gene mutations that affect the occurrence of NSCLC in secondhand smoke are exons 18 (G719X), 19 (deletions or insertions), and 21 (L858R and L861Q),32 while exon 20 (insert) mutations make up $4-10 \%$ of all EGFR mutations. Most of these mutations are located near the ends of the C-helix in the $\mathrm{N}$-lobe of kinases. ${ }^{31,32}$

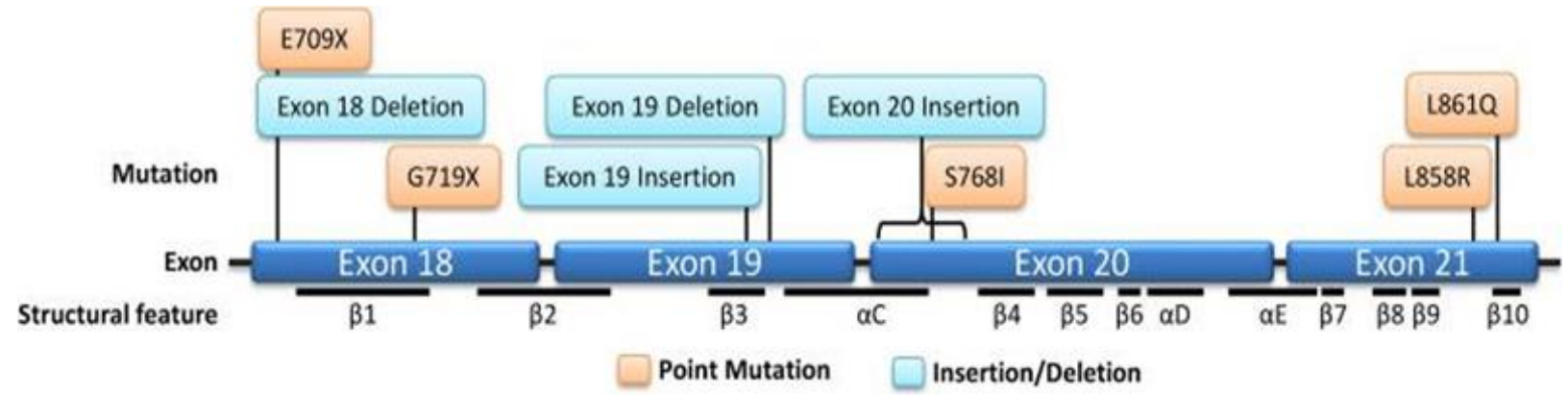

Figure 2. Location of the EGFR mutation. ${ }^{31}$

Mutation EGFR in NSCLC, in the form of the G719X substitution (which includes the G719S, G719A, G719C, and G719D substitutions) is one of the most common mutations observed after exon 20 insertions and represents about 1.5-3\% of all mutations EGFR in NSCLC. A low prevalence of exon 19 insertions and deletions has been reported in approximately $2 \%$ of exon 19 aberrations and 1\% of all EGFR mutation in NSCLC. The insertion of exon 19 is characterized by 18 base pairs resulting in the addition of 6 amino acid sequences with most lung cancer cases starting at codon 744 or 745 of the EGFR gene. ${ }^{30,31}$

\section{The Relationship of the EGFR gene to the occurrence of lung cancer}

The EGFR gene has an important role in cancer. The amplification and mutation of these genes are a driving factor for research and therapy in many types of cancer. EGFR mutations were detected in the normal respiratory epithelium of $43 \%$ of patients with lung adenocarcinoma and occurred in early lesions, such as atypical adenomatous hyperplasia. ${ }^{30,33}$ 


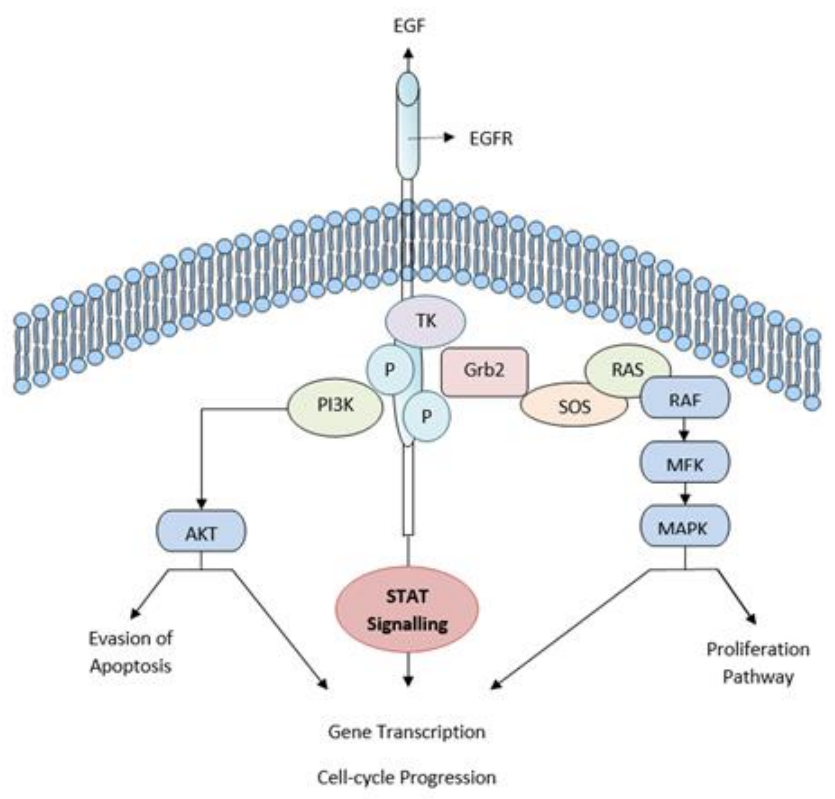

Figure 3. EGFR gene pathway in NSCLC

The epidermal growth factor receptor (EGFR) pathway has a ligand called epidermal growth factor (EGF) or transformation growth factor (TGF-a) that binds to homodimeric and heterodimeric tyrosine kinase domains to produce receptor activation and transphosphorylation, thereby creating a docking site for the adapter protein. Grb2 and Sos will recruit Ras and phosphatidylinositol 3-kinase (PI3K) to regulate the formation of two major signaling pathway branches via MAPK and PI3K causing these tissues to produce proliferation and decrease apoptosis. ${ }^{33}$

The literature study explained that EGFR gene mutations were significantly related to sample type, pathological type, lung cancer stage, and treatment method. The exon 18 mutation was mainly detected in stage IA NSCLC tissue samples. However, there was no significant difference between exon 19 and exon 20 mutational status with clinical indicators. The positive exon 19 mutation rate was higher in stage IB NSCLC patients, whereas the positive exon 20 mutation rate was higher in stage IIIB NSCLC patients. ${ }^{34}$

\section{EGFR tyrosine kinase inhibitor in lung cancer}

Protein kinases play an important role in signal transduction, cellular proliferation, differentiation, and other regulatory mechanisms. The identification of growth-associated protein kinases, particularly tyrosine kinases as therapeutic targets for cancer, and the ATP-binding domains of tyrosine kinases as targets used for drug design have resulted in the clinical development of a range of tyrosine kinase inhibitors (TKIs) in various malignancies, including lung cancer. In recent years, the clinical application of TKI to NSCLC as a heterogeneous disease with multiple genetic subsets has led to extended median survival of 3 years. 35

The EGFR protein is expressed in about $50-90 \%$ of cases of NSCLC. The two main approaches to EGFR inhibitors are the use of small-molecule inhibitors of the intracellular tyrosine kinase domain and monoclonal antibodies that block the extracellular domain of the receptor. Gefitinib and erlotinib are orally administered EGFR-TKIs that can compete with ATP for binding to the TK domain. 35 Uncommon EGFR mutation, such as G719X, L861Q, S768I and E709X are widely sensitive to EGFR-TKI, especially second and third generation agents. 36 


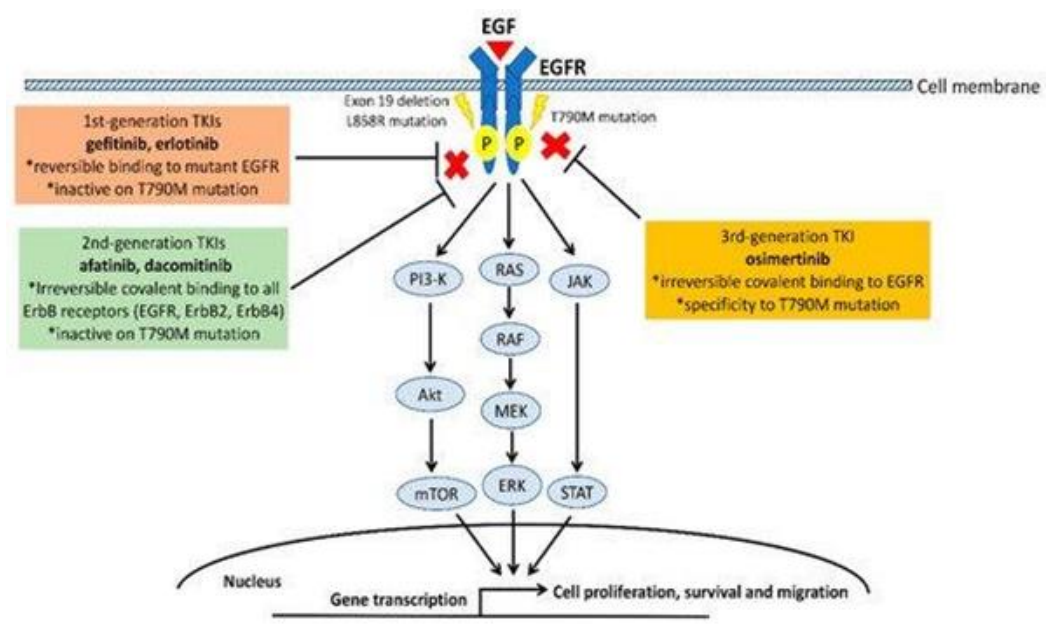

Figure 4. EGFR pathway to TKIs in NSCLC. 37

NSCLC harboring an EGFR L858R deletion mutation or exon 19 is sensitive to EGFR-TKIs, such as gefitinib, erlotinib, afatinib, dacomitinib, and osimertinib. Gefitinib and erlotinib are classified as first-generation EGFR-TKIs. Afatinib and dacomitinib are second-generation, and osimertinib is third generation. ${ }^{38}$ Gefitinib and erlotinib first-generation EGFR-TKIs have binding to the EGFR mutant but are not active against the T790M mutation. The secondgeneration EGFR-TKIs, namely afatinib and dacomitinib, have irreversible covalent binding to all ErbB receptors (EGFR heterodimers, ErbB2, ErbB4, and ErbB), and are inactive against the T790M mutation. The third-generation EGFR-TKI osimertinib has irreversible covalent binding to the EGFR mutant and is active in the T790M mutation. ${ }^{37,39}$

Clinical trials report that first and secondgeneration EGFR-TKIs produce an effective response of approximately $60-80 \%$ higher than conventional chemotherapy in NSCLC patients with EGFR mutations. The therapy can target missense and inframe mutation exons 18-21 in the TK domain of EGFR that significantly contribute to non-small cell lung cancer progression and metastasis. However, erlotinib and gefitinib were unable to target the mutation of threonine to methionine at codon 790 of exon 20 in the EGFR gene. Erlotinib and gefinib resistance are major problem in patients on long-term treatment and after relapse. EGFR-TKIs resistance usually occurs due to mutations in threonine to methionine codon 790 at the exon 20 site of EGFR or called mutation T790M. Patients with these mutations may be given therapy that mediates EGFR inhibition with a binding site away from the codon site. 36

T790M mutations account for $30-60 \%$ of secondary EGFR point mutations in NSCLC patients who are resistant and do not respond to therapy for first- and second-generation EGFR-TKIs.40 Clinical trial evidence shows $70 \%$ of osimertinib which is an EGFRTKIs effective in treating NSCLC patients with the T790M mutation. In addition, a phase 3 clinical trial demonstrated that osimertinib resulted in significantly longer survival than gefitinib and erlotinib in advanced NSCLC with the EGFR mutation. ${ }^{36}$

\section{Conclusion}

The EGFR gene is expressed by $60 \%$ in NSCLC and plays an important role in the processes of proliferation, survival, motility, and differentiation of tumor cells. Mutations in the TK domain of EGFR result in constitutive and oncogenic activation of receptors leading to tumor cell dependence in the EGFR signaling pathway. EGFR mutations in lung cancer occur at 
multiple sites, namely exons 18 to 21 EGFR. Mutations at exon 19 deletion and L858R point at exon 21 represented $87 \%$ of all EGFR mutations observed in NSCLC patients from secondhand smoke which were also associated with lung cancer stage, gender, sample type, and treatment modality. EGFR mutation analysis by direct sequencing is the most studied and reliable method for predicting response to therapy. However, an increase in the number of copies of the EGFR gene can be seen because the mutation is the initial event of lung cancer carcinogenesis followed by gene amplification. Thus, EGFR mutations and gene amplification status are important in determining the type of lung cancer that will respond to EGFR-TKI therapy.

\section{References}

1. Indonesian Ministry of Health. Info data and information on the general situation of tobacco consumption in Indonesia. 2018; 2-12.

2. Indonesian Ministry of Health. Riskesdas Result Report. 2018; 324-42.

3. Bade BC. Lung Cancer 2020: Epidemiology, etiology, and prevention. Vol. 41, Clinics in Chest Medicine. 2020; 1-24.

4. International Agency for research on cancer (IARC). Indonesia - Global Cancer Observatory. Globocan. 2020; 858: 1-2.

5. Kauffmann-Guerrero D. Pathology and diagnostics of lung cancer. Tagliche Praxis. 2020; 62: 64-71

6. Travis WD. Lung cancer pathology: Current Concepts. Clinics in Chest Medicine. 2020; 41 : 67-85.

7. ALQudah MA. Epidemiology and histopathological classification of lung cancer: A study from Jordan, retrospective observational study. Ann Med Surg. 2021; 65.

8. Altorki NK, Markowitz GJ, Gao D, et al. The lung microenvironment: an important regulator of tumor growth and metastasis. Nat Rev Cancer. 2019; 19(1): 9-31.

9. Girard N. Optimizing outcomes and treatment sequences in EGFR mutation-positive nonsmall-cell lung cancer: Recent updates. Vol.
15, Future Oncology. 2019. p. 2983-97.

10. Harrison PT. Rare epidermal growth factor receptor (EGFR) mutations in non-small cell lung cancer. Vol. 61, Seminars in Cancer Biology. 2020; 167-79.

11. Nurhasana R. Passive smokers' support for stronger tobacco control in Indonesia. Int $\mathrm{J}$ Environ Res Public Health. 2020; 17(6).

12. World Health Organization. Heart disease and stroke are the commonest ways by which tobacco kills people. World Healing Organs. 2018; 1(1): 1-2.

13. IAKMI T. Atlas of Indonesian Tobacco Year 2020. 2020; 33.

14. Linardatou V. Passive smoking acutely affects the microcirculation in healthy non-smokers. Microvasc Res. 2020; 128.

15. Zavala-Arciniega L. Correlates of second-hand smoke exposure in public and private settings in Mexico. Encodat 2016. Salud Publica Mex. 2019; 61(5): 591-600.

16. Doukas SG, Vageli DP, Lazopoulos G, Spandidos DA, Sasaki CT, Tsatsakis A. The Effect of NNK, A Tobacco Smoke Carcinogen, on the miRNA and Mismatch DNA Repair Expression Profiles in Lung and Head and Neck Squamous Cancer Cells. Cells. 2020; 9

17. Lu L, Liang Q, Shen S, Feng L, Jin L, Liang ZF. Tobacco Smoke Plays an Important Role in Initiation and Development of Lung Cancer by Promoting the Characteristics of Cancer Stem Cells. Cancer management and research. 2020; 12; 9735-9.

18. Butler KM, Huntington-Moskos L, Rayens MK, Wiggins AT, Hahn EJ. Perceived synergistic risk for lung cancer after environmental reportback study on home exposure to tobacco smoke and radon. American Journal of Health Promotion. 2019; 33(4): 597-600.

19. Pavlisko EN. Lung cancer: Clinical findings, pathology, and exposure assessment. Occupational Cancers. 2020; 205-26.

20. Du Y. Lung cancer occurrence attributable to passive smoking among never smokers in 
China: A systematic review and meta-analysis. Transl Lung Cancer Res. 2020; 9(2): 204-17.

21. Tang A. Non-small cell lung cancer in neverand ever-smokers: Is it the same disease? $\mathrm{J}$ Thorac Cardiovasc Surg. 2021; 161(6): 190317.

22. Arnal-Estapé A. Preclinical models for the study of lung cancer pathogenesis and therapy development. Cold Spring Harb Perspect Biol. 2021; 13(11).

23. Sister DI. Molecular pathology of primary nonsmall cell lung cancer. Vol. 51, Archives of Medical Research. 2020; 784-98.

24. Brosseau S. Lung cancer in nonsmokers. Rev Prat. 2020; 70(8): 851.

25. Yoneda K. Treatment of non-small cell lung cancer with EGFR-mutations. Vol. 41, Journal of UOEH. 2019; 153-63.

26. Vyse S. Targeting EGFR exon 20 insertion mutations in non-small cell lung cancer. Vol. 4, Signal Transduction and Targeted Therapy. 2019.

27. Yang G. EGFR exon 20 insertion mutations in Chinese advanced non-small cell lung cancer patients: Molecular heterogeneity and treatment outcome from nationwide real-world study. Lung Cancer. 2020; 145: 186-94.

28. Martin-Fernandez ML, Clarke DT, Roberts SK, Zanetti-Domingues LC, Gervasio FL. Structure and dynamics of the EGF Receptor as Revealed by Experiments and Simulations and Its Relevance to Non-Small Cell Lung Cancer. Cells. 2019 Apr 5; 8(4): 316.

29. Chang S. Molecular biomarker testing for nonsmall cell lung cancer: Consensus statement of the Korean Cardiopulmonary Pathology Study Group. Journal of Pathology and Translational Medicine. 2021; 55; 181-91.

30. Ercelep Ö Smokers having activating EGFR mutant non-small cell lung cancer might benefit from EGFR-TKI treatment - singlecenter experience. Turk Onkol Derg. 2020; 35(3): 334-9.

31. Harrison PT, Vyse S, Huang PH. Seminars in cancer biology rare epidermal growth factor receptor (EGFR) mutations in non-small cell lung cancer. Semin Cancer Biol. 2020; 167-79.

32. Matsui T, Tanizawa Y, Enatsu S. [Exon 19 Deletion and Exon 21 L858R point mutation in EGFR Mutation-Positive Non-Small Cell Lung Cancer]. Gan to kagaku ryoho. Cancer \& chemotherapy. 2021; 48; 673-6.

33. Lv P. Pathogenesis and therapeutic strategy in platinum resistance lung cancer. Vol. 1876, Biochimica et Biophysica Acta - Reviews on Cancer. 2021.

34. Li S, Li X. Analysis of EGFR, KRAS, and PIK3CA gene mutation rates and clinical distribution in patients with different types of lung cancer. World journal of surgical oncology. 2021; Vol. 19: 197.

35. Greenhalgh J. First-line treatment of advanced epidermal growth factor receptor (EGFR) mutation positive non-squamous non-small cell lung cancer. Cochrane Database System Rev. 2021; 2021(3).

36. Kim ES, Melosky B, Park K, Yamamoto N, Yang JC-H. EGFR tyrosine kinase inhibitors for EGFR mutation-positive non-small-cell lung cancer: outcomes in Asian populations. Future oncology (London, England). 2021; 17: 2395408.

37. Lim, SM, Syn, NL, Cho, BC, Soo, RA Acquired resistance to EGFR targeted therapy in nonsmall cell lung cancer: Mechanisms and therapeutic strategies. Cancer Treat. Rev.2018; 65: 1-10.

38. Shah R. Tyrosine kinase inhibitors for the treatment of EGFR Mutation-Positive NonSmall-Cell Lung Cancer: A Clash of the Generations. Clinical Lung Cancer. 2020; 21.

39. Aziz MIA, Foo WYX, Toh CK, Lim WT, Ng K. Cost-effectiveness analysis of osimertinib for first-line treatment of locally advanced or metastatic EGFR mutation positive non-small cell lung cancer in Singapore. Journal of medical economics. 2020; 23; 1330-9. 
40. Rui M, Li H. Cost-effectiveness of Osimertinib vs Docetaxel-bevacizumab in Third-line Treatment in EGFR T790M Resistance Mutation Advanced Non-Small Cell Lung Cancer in China. Clinical therapeutics. 2020; 42; 2159-70. 